Received by OSTI

LBL-25047

Fetor 1900

LB Lawrence Berkeley Laboratory UNIVERSITY OF CALIFORNIA

Accelerator \& Fusion Research Division

Presented at the Eighth Topical Meeting on

Technology of Fusion Energy, Salt Lake City, UT,

October 9-13, 1988

Heavy Ion Induction Linac Drivers for Inertial Confinement Fusion

E.P. Lee and J. Hovingh

October 1988

Prepared for the U.S. Department of Energy under Contract Number DE-A C03-76SF00098.

MASTER

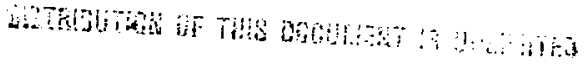




\title{
DISCLAIMER
}

This report was prepared as an account of work sponsored by an agency of the United States Government. Neither the United States Government nor any agency thereof, nor iny of their employees, makes any warranty, express or implied, or assumes any legal liability or responsibility for the accuracy, completeness, or usefulness of any information, apparatus, product, or process disclosed, or represents that its use would not infringe privately owned rights. Reference herein to any specific commercial product, process, or service by trade name, trademark. manufacturer, or otherwise does not necessarily constitute or imply its endorsement, recommendation, or favoring by the United States Government or any agency thereof. The views and opinions of authurs exprestod nerein do not necessarily state or reflect those of the United States Government or any agency thereof.

\section{Heavy Ion Induction Linac Drivers for Inertial Confinement Fusion*}

\author{
E.P. Lee \\ Accelerator and Fusion Research Division \\ Lawrence Berkeley Laboratory \\ 1 Cyclotron Road \\ Berkeley, California 94720
}

and

J. Hovingh

Lawrence Livermore National Laboratory

University of Califomia

Livermore, California 94550

October 1988

* This work supported by the Office of Energy Research, Office of Program Analysis, and Office of Basic Energy Sciences, U.S. Dept. of Energy under Contract No.

DE-AC03-76SF00098. 
LBL -25047

HIFN-388

\section{HEAVY ION INDUCTION LINAC DRIVERS FOR INERTLAL CONFINEMENT FLSION* *}

\author{
E. P. LEE \\ Lawrence Berkeley Laboratory \\ University of California \\ Berkeley, CA 94720 \\ (415) $486-7345$
}

\author{
J. HOVDGH \\ Lawrence Livennore National Laboratory \\ University of Califormia \\ Livermore, CA 94550 \\ (415) +22.5421
}

\begin{abstract}
Intense beams of high energy heavy ions (e.g. $10 \mathrm{GeV} \mathrm{Hg)} \mathrm{are} \mathrm{an} \mathrm{altractive} \mathrm{option} \mathrm{for} \mathrm{an} \mathrm{ICF}$ driver because of their favorable energy deposition characteristics. The accelerator systems to produce the beams at the required power level are a development from existing iechnologies of the induction linac, if linac/storage ring, and synchrotron. The high repecition rate of the accelerator systems, and the high efficiency which can be realized at high current make this approach especially suitable for commercial ICF. The present repor: gives a summary of the main fearures of the induction linac driver system, which is the approach now pursued in the USA. The main subsystems, consisting of injector, multiple beam accelerator at low and high energy, transport and pulse compression lines, and final focus are described. Scale relations are give for the current limits and other features of these subsystems.
\end{abstract}

\section{INTRODUCTION}

Inertial confinement fusion (ICF) requires very high power irradiance and energy desposited on the fusion target which are nearly independent of the driver type. Lit addition, the depth of deposition must be small (typically - 0.1 $g / \mathrm{cm}^{2}$ in a stopper material) to produce the high fusion yields required for an economically attractive power plant. The range condition can be met, in principle, by any ion species accelerated sufficiently to match the range-energy relation. The large stopping power for heavy ions in matter allows the use of kinetic energies

*This work supported by the Office of Energy Research, Office of Program Analysis, and Office of Basic Energy Sciences, U.S.D.O.E. Contract No. DE-AC03-76SF00098. in the range of $5.20 \mathrm{GeV}$. Required particle currents are therefore very low compared with those for light ions or phoions, but they are high compared with those usually associated with ion acceleration. Two conventional, but poteltatly high current. accelerator technologies are heing explored. These are the of linac/storage ring system now studied in W. Germany, the USSK and Japan and the induction linac approach of the USA. The induction linac, which is described here, appears to be well matched to the requirements of a power plant driver. It is expected to have high electrical efficiency $(\geq$ $20 \%$ ), high rep rate $(>>1 \mathrm{~Hz})$ and the very long operating life typical of conventional multigap accelerators.

A typical set of final beam parameters suituble for a power reactor are given in Table l. It must be emphusized that cost tradeoffs among the many components of a complete power plant allow a broad range of system parameters isuch as repetition rate) to be considered, with minor effect on the final cost of elecaicity (COE). The tabulated ciriver parameters are matched to a 1000 MWe plant with COE of about $60 \mathrm{mil} / \mathrm{k}$ Wh, and total direct capital cost of 2.2 G\$ $(1984 \$),(1,2)$ The driver contributes $43 \%$ of the cost in this case. While the COE is about double that available from existing, on-line coal or fission plants, it is comparable with the estimates from other fusion system studies (e.g. $59.1 \mathrm{mil} / \mathrm{kWh}$ for the 120 MWe STARFIRE tokamak in 1984 dollars using current costing methods). The primary concern at present is not so much the COE but the magnitude of generating capacity and capital investment of the plant. A 500-1000 MWe fusion plant with the stated $C O E$ is of considerable interest, but it is difficult to achieve, primarily because of the economy of scale associated with all nuclear electric plants. Both the If linac/storage ring and the induction linac drivers provide a substantial fraction of total 
direct capital costs of a plant and scale poorly for lower net electric power. Magnetic fusion systems are also very large for reasons of economy of scale as well as physical constraints imposed by the use of low density plasma.

Table 1

Heavy Ion Beam Drivers Parameters

\begin{tabular}{ll}
\hline Pulse energy & $4.0 \mathrm{MJ}$ \\
Paiticle energy & $10.0 \mathrm{GeV}$ \\
Particle type & $\mathrm{Hg}(\mathrm{A}=200)$ \\
Peak poiver & $400 \mathrm{TW}$ \\
Pulse length & $10 \mathrm{~ns}$ \\
Rep. rate & $10 \mathrm{~Hz}$ \\
Number of beims & 16 \\
Net pulse charge & $1200 \mu \mathrm{c}$ \\
Relativistic factor ( $\beta \gamma)$ & .332 \\
Emimince (unnomalized) & $3 \times 10^{-5} \mathrm{~m}-\mathrm{r}$ \\
Momentuni width & $\pm .1 \%$ \\
Spot radius & $4 \mathrm{~mm}$ \\
Convergence half angle & $15 \mathrm{mr}$ \\
Standotf to tinal magnet & $10 \mathrm{~m}$ \\
Target gain & 80 \\
Net electric power & $1000 \mathrm{WW}$ \\
\hline
\end{tabular}

The present report gives a summary of the main fearure of the induction linac driver system. The main subsystems, consisting of injector, multiple beam accelerator at low and high energy, uransport and pulse compression lines and final focus are described. Scale relations are given for the current limits and other features of these subsystems.

\section{GENERAL FEATURES OF THE INDUCTION LLNAC SYSTEM}

An induction linac driver is now envisioned as a multiple beamlet transport lattice consisting of $V$ closely packed parallel FODO transport channels. Each tocusing channel is composed of a periodic system of tocusing $F$ and detocusing $D$ quadrupole lenses with drift spaces $O$ between successive lenses. Surrounding the transport structure are massive induction cores of fertomagnetic material and associated pulse curcuitry that apply a succession of long duration. high-voltage pulses to the $N$ parallel beamlets. Longitudinal tocusing is also achieved through the detailed timing and shape of the accelerating walveiorms (with icedback correction of errors).
A multiple beam source of heavy ions operates at 2 to $3 \mathrm{MV}$, producing the net charge per pulse required to achieve the desired pellet gain. Initial current and, therefore, initial pulse length are determined by transpor limits at low energy. The use of a large number of electrostatic quadrupole channels $(N \sim 16$ to 64$)$ appears to be the least expensive focal option at low energies (below $-50 \mathrm{MV}$ ). This is tollowed by a lower number of superconducting tnagnetic channels $(N \sim 4$ to 16) for the rest of the accelerator. Merging of beams may therefore be required at this transition. Furhermore, some splitting of bearns mily be required after acceleration to stay within current limits in the tinal tocus system.

The rationale for the use of multiple beams is that it increases the net charge that can be accelerated by a given cross section of cure at a fixed accelerating gradient. Altematively, a given amount of charge can be accelerated more rapidly with multiple beams since the pulse length is shortened and a core cross section of specified volt-seconds per metre tlux-swing can supply an increased gradient. However, an increase in the number of beamlets increases the cost and dimensions of the transport lattice and also increases the cost of the core for a given voltsecond product since a larger core volume is required. For a core of given cross sectional area ( $\alpha$ voli-seconds per metre), the volume of ferromagnetic material increases as its inside diameter is increased. Hence, there is a trade-otf between transport and acceleration costs with an optimum at some finite number of beamlets. The determination of this optimum contiguration is a complex problem depending on projected costs of magnets, core insulators, energy storage, pulsers, and fabrication.

The choice of superconducting magnets for the bulk of the linic is mandated by the requirement of system efticiency: this must be at least $\sim 10 \%$ in an ICF driver and ideally $\geq 20 \%$ to avoid large circulation power fractions which result in a high cost of electricity). Induction cores are most likely to be constructed trom thin laminations if amorphous iron, which is the preferred material due to its excellent electrical characteristics and flux-swing. At a projected cost of approximateiy $\$ 8.3 / \mathrm{kg}$ (insulated and wound), this is a major cost item for the tirst 2 to $3 \mathrm{GV}$ of a typical linac. At higher voltage, the 
cost of pulses and fabrication of the high gradient column with insulators dominates.

Between the accelerator and the fusion reactor, the beamlets are separated radially in space and, if necessary, split with a kicker and magnetic septum. The drift lines leading to the final focus area are 200 to $600 \mathrm{~m}$ long and used for ballistic compression as well as to march the final focus configuration of the reactor. The transport lattice is composed of cold bore superconducting quadrupoles, bends, and possibly higher order elements needed to control momentum dispersion. As the beamlets compress, the rransport of the high current becomes increasingly demanding, with the large apertures and the close packing of elentents especially pronounced immediately before the final focus train.

The final focus system itself has paramelers determined largely by the requirements of spot size on target, reactor size, and the handling of neutron, $X$-ray, and gas fluxes from the reaclor. The final focus magnet train is composed of six or more magnetic quadrupoles of large bore and several weak bends used to remove line-of-sight neutrons. Its total length is 50 to $100 \mathrm{~m}$ per beamline.

Transport within the reactor vessel has, in most studies, been assumed to take place in nearvacuum ( $P \leq 10^{-4}$ Tor lithium) to avoid disruption by the two-stream instability, or in a high-pressure window $\left(P-10^{-1}\right.$ to 10 Torr). where the beam is also thought to be stable. 3 The HIBALL reactor specifies $P<10^{-5}$ Torr lend vapor to avoid stripping of beam ions, which would lead to reduced target irradiance due to the beam's electric field. Unfortunately, several atractive reactor concepts ICASCADE (Ref, 4), HYLIFE (Ref. 5)] have residual gas pressures in the range $10^{-2}$ to $10^{-3}$ Torr lithium at reasonible repetition rates; this pressure must be taken into account both for transport in the reactor and in maintaining vacuum in the final locus lines. Recent calculations 6 show that the two-stream mode is benign at these pressures due to the detuning effects of beam convergence.

Major component systems of the driver are respresented in Fig. 1

\section{Fig. 1: INDUCTION LINAC DRIVER $(A=200, q=3)$}

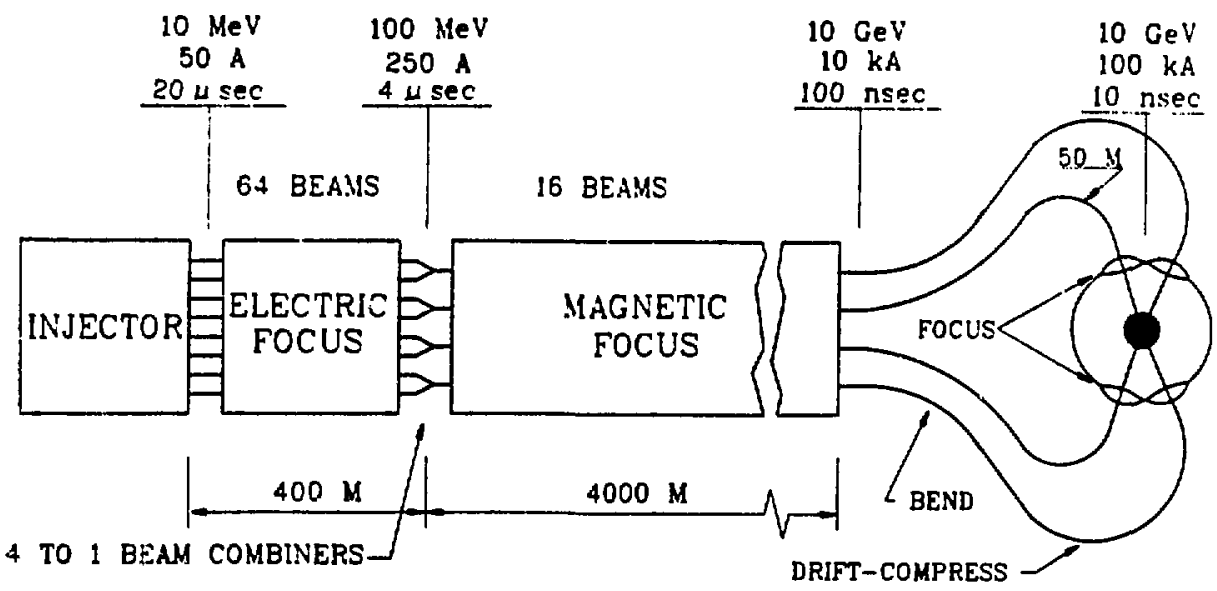

XBL 865-1965 A 
Multiple Beamlet Iniector

The ion source produces the required quantity of ions in the desired charge state, and injects them into the low-voltage section of the accelerator at a voltage high enough for efficient transport $(-3 \mathrm{MV})$. The maximum current density available from a planar diode limited by space-charge effects is given by the ChildLangmuir law?:

$$
\begin{aligned}
j & =\frac{4 \sqrt{2}}{g}\left(\frac{e}{m_{0} c^{2}} \frac{\varepsilon_{0}}{\mu_{0}} \frac{q}{A}\right)^{1 / 2} \frac{V_{s}^{3 / 2}}{d^{2}} \\
& =5.40 \times 10^{-3}\left(\frac{G}{A} \frac{V_{i}^{3}}{d^{4}}\right)^{1 / 2}\left(\mathrm{~N}^{2} \mathbf{H}^{2}\right),
\end{aligned}
$$

where

$$
\begin{aligned}
& V_{s}=\text { extractor voltage (typically }=100 \mathrm{kV} \text { ) } \\
& d=\text { source extraction gap width } \\
& q=\text { ion charge stale } \\
& m_{0}=\text { atomic mass unit. } \\
& A=\text { mass number }
\end{aligned}
$$

The normalized emittance $\pi \varepsilon_{n}$, which is the invariant transverse $\left(x, x^{\prime}\right)$ phase volume occupied by a beamlet, is determined by the source characteristics and injector optics. For an ideal injector having no aberations, the emittance cian be simply related to the source radius $a_{5}$ and iemperature $T_{s}$ according to

$$
\begin{aligned}
& E_{n} \equiv 4 \beta y\left(\overline{x^{2}} \overline{x^{2}}-\left(\overline{x x^{\prime}}\right)^{1 / 2}\right. \\
& =6.55 \times 10^{-5}\left(\frac{T_{s}}{A}\right)^{1 / 2} a_{s}(m \cdot r a d) .
\end{aligned}
$$

where $T_{S}$ is given in electron-volts.

From the basic Eqs. (1) and (2), the source sharacteristics and some fundamental limits on the parameters of injected heavy-ion beamlets can be inferted. Using large area diodes $\left(-30 \mathrm{~cm}^{2}\right)$, heilvy-ion curents in the range of 1 to 2 amperes with $\varepsilon_{n}=10^{-6}$ to $10^{-7} \mathrm{~m} \cdot \mathrm{rad}$ are plausible, although this capability has not yet been realized in practice. Currents of this magnitude are well matched to the low-energy linac transport capability, while the emittance is 1 to 2 orders lower than the limit imposed by final focus. Hence, there is ample latitude for emittance growth during acceleration and the various beam manipulations.

\section{Accelerator Sustem}

The acceleration of the ion beam takes place in the drift sections of the FODO lattaice. The linear induction accelerator is equivalent to a transformer with the beam acting as a single-turn secondary. A toroidal core of ferromagnetic material is excited by a primary winding trom a high-power pulser/modulator. Combining Faraday's law with Stokes' theorem, the change in the magnetic tlux in the core is accompanied by an electric field across an acceleration gap

$$
\int E \cdot d l=\cdot \int_{S} \frac{d B}{d t} \cdot d S \text {. }
$$

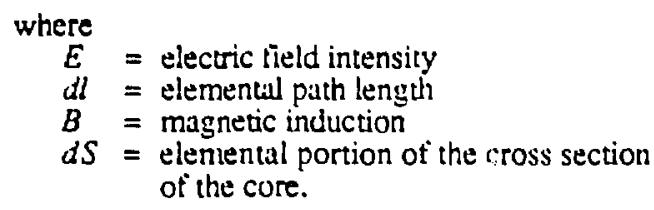

Since

$$
\int E \cdot d l=V_{c},
$$

where $V_{c}$ is the voltage applied to the core, Eq. (3) can be written as

$$
\tau V_{c}=S \Delta B \text {. }
$$

$$
\begin{aligned}
& \text { where } \\
& \begin{aligned}
S & =\text { cross-sectional area of the core } \\
& =\pi\left(r_{2}^{2}-r_{1}^{2}\right)
\end{aligned} \\
& \Delta B=\text { magnetic thux swing in the core } \\
& \tau=\begin{array}{l}
\text { temporal duration of the pulse } \\
\text { including the rise and fall time. }
\end{array}
\end{aligned}
$$

The essential role of the core is to permit a series of high-voltage pulses of up to tens of microseconds duration (instead of nanoseconds) to be applied to the bean at successive acceleration gaps. 
The acceleration system consists of a focal subsystem, the magnetic core subsystem as well as the modulator subsystem. Other major subsystems of the accelerator include the heat removal, beam alignment, control and diagnostics, insulation, supporting structure, and safety. A brief discussion of the major components follows.

The lens subsystem consists of electrostatic or magnetic lens sets. In general, the lens configurations may include quadrupoles, sextupoles, higher order multipoles, and solenoids. Magnetic solenoids, in principle, may allow higher current densities per beam than quadrupoles at low ion kinetic energies, but are not under consideration for low-energy transport at present due to a perceived economic disadvaniage. At moderate to high beam voltages, the quadrupoles clearly allow a higher current density than the solenoids, and have been used in most conceptual driver designs. The selection of quadrupoles over higher order multipoles is based in part on the linearity of the quadrupole fields, which is desired to conserve emittance. The focusing quadrupoles can be either electrostatic, or magnetic. Due to the factor of velocity in the magnetic force law, magnetic quadrupoles are the choice at high energy and electrostatic quadrupoles at low energy. In a typical conceptual HIF driver, the magnetic focusing system allows a higher beam current density than an electrostatic focusing system for voltages above some 10 to $100 \mathrm{MV}$. Several types of magnetic focusing quadrupoles can be used; for example, they can be either pulsed or steady devices. The pulsed quadrupoles and water-cooled steady-state electromagnets tend to be too inefficient for extensive use in a power plant. The steady-state magnetic quadrupole transport system has an option of using either permanent and/or superconducting magnets. The achievable pole-tip field strength for permanent magnets is, at the most, $-25 \%$ of that for superconducting magnets. This yields a transportable current density through a permanent magnet quadrupole set that is $<16 \%$ of that using a superconducting magnet quadrupole set of comparable dimensions, and makes them unattractive for most scenarios.

The vacuum required in the beamline is determined by the allowable beam losses from interaction of the beam ions with the residual background gases. The stripping cross sections tend to decrease with the ion kinetic energy, so the vacuum requirement is more severe at the low-voltage end of the accelerator. To keep the total beam losses from interaction with the background gas <5\%, the background gas number density must be $<10^{7}$ to $10^{9}$ particle $/ \mathrm{cm}^{3}$. These densities can be achieved in a well-designed system using turbomoleculiur or cryogenic pumps.

The acceletator cores can be fabricated from either dielectric or ferronagnetic material. Since the ferromagnetic material has a higher electrical impedance to the driving source than a dielectric, the ferroniagnetic cores are preferted. The cores are wound from thin tape, with insulation between the layers to allow for rapid fich penetration and to decrease the eddy current losses, which ideally scale as the lape thickness squared divided by resistivity. Several cores can be driven in parallel; utilizing either radial or longitudinal stacking arrangements to increase the acceleration gap voltage.

The power delivered to the cores is increased from that delivered by the primary energy source by a series of pulse energy compression steps. A power supply charges a pulse generator such as a Marx generator or pulse-forming network (which includes a high-power switch). The output from this modulator drives the load current, which is a parallel combination of the beam current (assumed constant during the pulse), and the cors currents, which increase during the pulse. A network consisting, for example, of a resistor and capacitor in series can be used to compensate for the increase in the core eddy current such that the total impedance of the core plus compensator is nearly constant.

\section{TRANSPORT}

In the absence of focusing, space-charge and emittance effects would cause the ion beam to expand radially. To control the transverse motion of the ions, lenses are used along the length of the driver and subsequent transport lines. For this study, these lenses, which are either electrostatic or magnetic quadrupoles, are aranged in a $F O D O$ (focusing-drift-defocusingdrift) periodic lattice. A simple set of scale formulas relates the principal parameters of a 
magnetic FODO lattice (average beam radius $\bar{a}$, field at average beam radius $B$, and half-period length $L$ ) to the principal beam parameters (electric current $I$, normalized emittance $\varepsilon_{n}$, and relativistic factor $B \gamma$ ). It is also necessary to specify the fraction of the lattice occupied by quadrupoles $\eta$, the phase advance per lattice period or tune $\sigma_{0}$, and the depressed value of the tune $\sigma$ resulting from the partial cancellation of the focal force by the beam's self-generated field. These relations may be cast into the approximate forms

$$
\begin{aligned}
& I=(2.39 \mathrm{MA})\left[1 \cdot\left(\frac{\sigma}{\sigma_{0}}\right)^{2}\right] \\
& \left.x \sigma_{u}^{\prime}(\beta \gamma)^{5} \eta^{2}\left(\frac{\mathrm{A}}{\mathrm{q}}\right)\left(\frac{\epsilon_{\mathrm{n}}}{\sigma}\right)^{2} \mathrm{~B}^{2}\right]^{1 / 3}, \\
& \overline{3}=(2.32 \mathrm{~m})\left[\left(\frac{\sigma_{0}}{\eta}\right)\left(\frac{\perp}{\beta \gamma}\right)\left(\frac{\mathrm{A}}{\mathrm{q}}\right)\left(\frac{\varepsilon_{\mathrm{n}}}{\sigma}\right)^{2}\left(\frac{1}{\mathrm{~B}}\right)\right]^{1 / 3},
\end{aligned}
$$

and

$$
\left.L=(2.68 \mathrm{~m}) \cdot\left(\frac{\sigma_{0}}{\eta}\right)^{2}(\beta \gamma)\left(\frac{A}{\mathrm{q}}\right)^{2}\left(\frac{\varepsilon_{\mathrm{n}}}{\sigma}\right)\left(\frac{1}{\mathrm{~B}}\right)^{2}\right]^{1 / 3},
$$

where $B$ is given in Tesla, $\varepsilon_{n}$ in metre-radians, and the tunes in radians per period. From Eqs. (6) and (7), the beamlet current density is

$$
\begin{aligned}
j= & \left(0.171 \frac{\mathrm{NA}}{\mathrm{m}^{2}}\right)\left[1 \cdot\left(\frac{\sigma}{\sigma_{1}}\right)^{2}\right] \\
& \left.\left.x^{\prime}\left(\sigma_{1}\right)\right)^{2} \eta^{4}(\beta \gamma)^{7}\left(\frac{\mathrm{q}}{\mathrm{A}}\right)\left(\frac{\bar{s}}{E_{n}}\right)^{2} B^{4}\right]^{1 / 3} .
\end{aligned}
$$

From Eq. (6), we anticipated that operation at low values of $\sigma$ results in high values of transportable current $I$. However, this strategy also results in large values of the beam radius $\bar{a}$ In general, a cost minimum can be tound, typically with $\sigma$ in the 8 - to $24-\mathrm{deg}$ range, depending on the number of beamlets, total charge accelerated, emittance, and, especially, ratio $q / A$.

Compression

At the end of acceleration, the ion pulse is typically 100 to $400 \mathrm{~ns}$ long, which is well matched to the bandwidth of the pulse forming system. Subsequent reduction to the desired 5 to 20-ns length desired for the fusion pellet impolsion dynamics is achieved by the mechanism of drift compression in the rransport lines leading to the final tocus system. If the initial pulse length (in metres) is $l_{0}$ and the drift lines have length $: 0$, then a head-to-tail velocity tilt (at a tixed time) of approximbitely

$$
\frac{\Delta V}{v}=\frac{l_{0}}{z_{0}}
$$

must be applied in the final stages of acceleration. It, for example, $l_{0}=20 \mathrm{~m}$ and $: 0=400 \mathrm{~m}$, then the pulse tail must move $5 \%$ taster than the head in the transport lines.

\section{Final Eocus and Transpor within Reacror}

To tocus the beamlets to a small spot radius (r) at the fusion pellet, a special final focus system is employed. It must convert the matched beam envelope of the transport lines into large radii with appropriate convergence angles at the chamber entrance. The tinal focus quadrupole triples described by Marin ${ }^{9}$ are well-suited as the basic beamline components and not described here.

The minimum number of tinal beanlines $N_{0}$ required to transport the beam ions to the tusion pellet with radius $r$ can be estimated from the consideration of space-charge effects in the reactor chamber. First, consider that the beumlets traverse the chamber in vacuum and that space-charge is the only detocusing eifect. Then the beam envelope equation is

$$
\frac{d^{2} a}{d s^{2}}=\frac{K_{0}}{3}
$$

where $K_{0}$ is the beamlet perveance

$$
K_{0}=\frac{2 I q e}{(\beta \gamma)^{3} m_{1} c^{3} A+\pi \varepsilon_{0}}=\frac{2 I q}{(\beta \gamma)^{3} A\left(31 \times 10^{6} A\right)} \text {. }
$$


The perveance is a dimensionless measure of beamlet current. The minimum beam radius resulting from Eq. (11) is

$$
r=a_{\text {lens }} \exp \left(-\theta^{2} / 2 K_{0}\right),
$$

where $\theta$ is the convergence cone half-angle and

$$
a_{\text {lens }}=L \theta
$$

is the beam radius at the final lens. For a power reactor, we expect standoif length $L=51010 \mathrm{~m}$, $\theta=10$ to $20 \mathrm{~m}$-rad, and $r=2$ to $4 \mathrm{~mm}$. To make space-charge negligible, we therefore require, in the absence of neurralization.

$$
K_{0} \leq(0.1) \theta^{2} .
$$

This condition leads to unacceptably large numbers of beamlets when the charge state exceeds $q=2$ to 4 , so some degree of neutralization must be invoked in general, either from the ionization of residual gas or coinjection of electrons. Recent calculations by Olson 10 indicate that the ion pulse is able to trap an electron cloud of sufficient density and low enough temperature to accomplish this. Thus, we allow $K_{0} \leq \theta^{2}$. The number of beamiets $N_{0}$ can be related to the total energy delivered to the pellet $W$ by

$$
\begin{aligned}
N_{0} & =\frac{W}{I_{p} T_{0} / q e}=\frac{4 W e^{2} q^{2}}{K_{0}(\beta \gamma)^{5} A^{2} m_{\gamma}^{2} c^{5} 4 \pi \varepsilon_{0} t_{p}} \\
& =(0.138)\left(\frac{q}{A}\right)^{2} \frac{W_{M J}}{K_{0}(\beta \gamma)^{5} t_{n s}},
\end{aligned}
$$

where final pulse length is $i_{p}$ (or ins in nanoseconds). For the typcal case ( $q=3, A=$ $200, W_{\mathrm{MJ}}=4, t_{n s}=10, K_{0}=2.25 \times 10^{-4}, \beta \gamma=$ 0.33 ), we get $N_{0} \geq 14.1$, which rounds up to $N_{0}$ $=16$ for symmetric two-sided illumination.

To produce a small radius $r$ on the target, the nomalized beamlet emittance $\varepsilon_{n}$ must satisfy

$$
\varepsilon_{n}<\beta y \theta \text {. }
$$

Allowance must also be made for the effect on spot size of momentum dispersion, various forms of jitter, and iesidual space-charge-induced blowup. A final focus system composed of quadrupoles and weak bends has momentum dispersion at the target which leads in a practical design based on a pair of triplets to increased spot radius

$$
\Delta r=\delta F \theta \frac{\Delta p}{p} .
$$

where $F$ is the distance from pellet to the center of the final quadrupole. Without compensition by high order elements, it is desirable to keep the momentum variation $\Delta p / p \leq 10^{\circ}$. This is at severe requirenent to be net by the accelerator system. Combining Eqs. (15) and (16), the spot radius on target is

$$
r=\left[\left(\frac{\varepsilon_{n}}{\beta \gamma \theta}\right)^{2}+6 i\left(F \theta \frac{\Delta p}{p}\right)^{2}\right]^{1 / 2} .
$$

Equations (1) لlurough (19) constitute a brief summary of the physics foundation of drivers for ICF based on linear induction accelerators. Further descriptions can be found in the literature. $11-17$

\section{REFERENCES}

1. D. DUDZIAK, W. SAYLOR, and W. HERRMANNSFELDT, "Fusion Technology," Vol. 13 No. 2, 207. Feb. (1988).

2. D. ZUKERMAN, D. DRIEMEYER, L. WAGANER, and D. DUDZIAK, jbid, 217.

3. C. L. OLSON, "Final Transport in Gas and Flasma," Proc. Heavy Ion Fusion Workshop, Berkeley, California, October 29-November 9, 1979, LBL-10301, Lawrence Berkeley Laboratory (1980).

4. J. H. PITTS and I. MAYA, "The Cascade Inertial-Confinement-Fusion Power Plant," Proc. Ilth Symp. Fusion Engineering, 
Austin, Texas, Novermber 18-22, 1985 , IEEE Cat. CH 2251-7, p. 103, Institute of Electrical and Electronics Engineers (1986).

5. J. A. BLINK, W. J. HOGAN, J. HOVINGH, W. R. MEIR, and J. $H$. PITTS, "The High-Yield Lithium-Injected Fusion-Energy (HYLIFE) Reactor," UCRL53559, Lawrence Livermore National Laboratory (1985).

6. P. STROUD, "Streaming Modes in Final Beam Transport lor Heavy Ion Beam Fusion," Laser Particle Beam, 4, Part 2, 261 (May 1986).

7. J. D. LAIVSON, The Physics of ChargedParticle Beams, Clarendon Press, Oxford (1977).

8. E. P. LEE, "Transport of Intense Ion Beams," Proc. 2nd Int. Conf. Charged Particle Optics, Albuquerque, New Mexico, May 19-23, 1986; see also LBL.21559, Lawrence Berkeley Laboratory (June 1986).

9. R. L. MARTIN, "Emittance Limitations in Heavy Ion Fusion," Nucl. Instrum. Methods, 187, 271 (1981).

10, C. L. OLSON, Heavy Ion Fusion, AIP Conference Proceedings. 152 (M. Reiser, T. Godlove, R. Bangerter, eds.) (AIP, New York 1986) p. 215.

11. D. KEEFE, "Research on High BeamCurrent Accelerators," Part. Accel. 11, 187 (1987).

12. S. HU.MPHRIES, Jr., Principles of Charged Particle Accelerators, Wiley Interscience Publishers. New York (1986).

13. R. C. ARNOLD. Eci., Proc. Heavy Ion Fusion Workshop, Argonne, Illinois, October 1979, ANL-79-41, Argonne National Laboratory (1979).

14. W. B. HERRRMANNSFELDT, Ed., Proc. Heavy Ion Fusion Workshop, Berkeley, Calitornia, October 29-November 9, 1979, LBL-10301, Lawrence Berkeley Laboratory (Sep. 1980).
15. T. J. FESSENDEN, "Induction Linacs for Heavy Ion Fusion Research," 'Proc. Linear Accelerator Conf., Seeheim/Darmstadt, FRG, September 1984.

16. D. BÖHNE, Ed., Proc. Symp. Accelerator Aspects of Heavy Ion Fusion, Darmstadt, FRG, GSI-82-8, Gesellschaft für Schwerionenforschung (Mar. 1982).

17. Y. HIRAO, T. KATAYAMA, and N. TOKUDA, Eds., Proc. INS Int. Symp. Heavy Ion Accelerators and Their Application to Inertial Fusion, Tokyo, Japan, Jan. 23-27, 1984, Institute for Nuclear Study, University of Tokyo (1984). 